\title{
Spectral and temporal gating mechanisms enhance the clutter rejection in the echolocating bat, Rhinolophus rouxi
}

\author{
Ingrid Neumann and Gerd Schuller* \\ Zoologisches Institut der Ludwig-Maximilian Universität München, W-8000 München 2, Federal Republic of Germany
}

Accepted April 29, 1991

Summary. Doppler shift compensation behaviour in horseshoe bats, Rhinolophus rouxi, was used to test the interference of pure tones and narrow band noise with compensation performance. The distortions in Doppler shift compensation to sinusoidally frequency shifted echoes (modulation frequency: $0.1 \mathrm{~Hz}$, maximum frequency shift: $3 \mathrm{kHz}$ ) consisted of a reduced compensation amplitude and/or a shift of the emitted frequency to lower frequencies (Fig. 1).

Pure tones at frequencies between 200 and $900 \mathrm{~Hz}$ above the bat's resting frequency (RF) disturbed the Doppler shift compensation, with a maximum of interference between 400 and $550 \mathrm{~Hz}$ (Fig. 2). Minimum duration of pure tones for interference was $20 \mathrm{~ms}$ and durations above $40 \mathrm{~ms}$ were most effective (Fig. 3). Interfering pure tones arriving later than about $10 \mathrm{~ms}$ after the onset of the echolocation call showed markedly reduced interference (Fig. 4). Doppler shift compensation was affected by pure tones at the optimum interfering frequency with sound pressure levels down to $-48 \mathrm{~dB}$ rel the intensity level of the emitted call (Figs. 5, 6).

Narrow bandwidth noise (bandwidth from $\pm 100 \mathrm{~Hz}$ to $\pm 800 \mathrm{~Hz}$ ) disturbed Doppler shift compensation at carrier frequencies between $-250 \mathrm{~Hz}$ below and $800 \mathrm{~Hz}$ above RF with a maximum of interference between 250 and $500 \mathrm{~Hz}$ above resting frequency (Fig. 7). The duration and delay of the noise had similar influences on interference with Doppler shift compensation as did pure tones (Figs. 8, 9). Intensity dependence for noise interference was more variable than for pure tones $(-32 \mathrm{~dB}$ to $-45 \mathrm{~dB}$ rel emitted sound pressure level, Fig. 10).

The temporal and spectral gating in Doppler shift compensation behaviour is discussed as an effective mechanism for clutter rejection by improving the processing of frequency and amplitude transients in the echoes of horseshoe bats.

Abbreviations: $C F$ constant frequency; $F M$ frequency modulation; $R F$ resting frequency; $S P L$ sound pressure level

* To whom offprint requests should be sent
Key words: Rhinolophus rouxi Doppler shift compensation - Temporal gating - Spectral gating - Clutter rejection

\section{Introduction}

Echolocating bats are active in different environments. Bat species which catch prey in forests or jungles will have to cope with cluttering echoes reverberated from the dense background such as foliage, twigs, branches, etc. The socalled Doppler shift compensation behaviour of horseshoe bats, which is explained below, is a good paradigm to study the influence of interfering signals on echolocation.

The Sri Lankan rufous horseshoe bat, Rhinolophus rouxi, catches flying insects in the jungle on the wing or with a hang-and-wait strategy (Neuweiler et al. 1987). While searching for prey the horseshoe bats emit long (20-60 ms) echolocation signals which consist of a pure tone component $(\mathrm{CF})$ of an individual frequency called resting frequency (RF) within a species-specific frequency range of $72-79 \mathrm{kHz}$. This component functions as a carrier for frequency modulations which are superimposed on the CF tone when it is reflected from the beating wings of insects or other moving targets. These modulations characterize a flying prey as moving target to the echolocating horseshoe bat.

The auditory system of the horseshoe bats has a disproportionally large population of neurons which are narrowly tuned to an individual 'reference frequency'. The reference frequency is up to $300 \mathrm{~Hz}$ higher than the resting frequency (RF) emitted by a nonflying horseshoe bat.

The echolocation frequency and the small frequency band of extremely narrowly tuned neurons are closely matched in each specimen (Schuller 1980; Schuller and Pollak 1979). However, when the horseshoe bat is flying the frequency of the echoes will be shifted to higher frequencies due to Doppler effects. The flying horseshoe 
bat avoids mismatches between the echo frequency and the reference frequency by lowering the frequency of subsequently emitted signals by an appropriate amount. This audiovocal feedback behaviour is called Doppler shift compensation and stabilizes the echo frequency at the reference frequency (Schnitzler 1968; Schuller et al. 1974).

Doppler shift compensation behaviour depends on the detection of frequency disparity between the echo frequency and the reference frequency of the bat's auditory system. Under natural conditions Doppler shift compensation behaviour, as well as any other echo processing, is exposed to interfering signals. A spatially and temporally distributed array of echoes reflected from foliage and other background structures will interact with the echoes reflected from a putative prey. In order to successfully track a flying insect under such cluttering conditions the bat will have to develop strategies to overcome cluttering interferences.

Several strategies are effective and may be used with varying priorities in different bat species and for distinct echolocation tasks:

a) Directionality of sound emission and sound reception cuts out a private spatial cone for the individual bat (Schnitzler and Grinnell 1977; Grinnell and Schnitzler 1977) and considerably reduces the interference of cluttering echoes from outside the relevant spatial area.

b) Individual spectral signature of echolocation calls allows the bat to distinguish them from 'foreign' echoes from other individuals.

c) The relevant temporal delay of echoes to the echolocation call emission is determined by the spatial depth or range within which the bat hunts. Thus only echoes reaching the bat's ear within this 'temporal window' will be identified as its own echoes. Temporal gating mechanisms have been demonstrated in several bat species (Schuller 1974, 1977; Roverud and Grinnell 1985ac; Roverud 1989a, b).

d) Neural mechanisms might reduce the influence of broad band noise from the environment, thus enhancing the contrast of the usefull echoes (Engelstätter et al. 1980).

The Doppler shift compensation behaviour in horseshoe and moustached bats is based on an audiovocal feedback system with a slow response time, and it only responds to frequency shifts above the resting frequency (RF) (Schuller et al. 1975). Its performance is closely linked to the onset of vocalization by a temporal gating mechanism and it does not react to auditory stimuli occurring during interpulse pauses of echolocation sound emission (Schuller 1974, 1977). During long pauses of vocalisation the lowering of the emission frequency is preserved and only updated when the bat emits subsequent echolocation calls (Schuller and Suga 1976).

The aim of this study was to test the resistance of the Doppler shift compensation system to interfering signals during echolocation. The Doppler shift compensation is a very convenient behaviour for evaluating quantitatively the performance of auditory echo frequency processing in the presence of cluttering noise.

\section{Materials and methods}

Three bats, Rhinolophus rouxi (two females from Sri Lanka and one male from India) were used for the Doppler shift compensation experiments. Those specimens which most frequently emitted echolocation calls when sitting in the playback apparatus were selected from a larger sample. These bats also consistently compensated for Doppler shifts introduced in the echoes by the Doppler shift simulation device. The resting frequencies of the bats were 75.9, 78.9 and $80.9 \mathrm{kHz}$, respectively.

The experiments were performed in the middle of a small room. The minimum distance of the bat from the surrounding walls and the floor was $1.5 \mathrm{~m}$. The bat was fixed in its normal hanging posture in an animal holder ('sandwich') that prevented wing movements but did not otherwise impair its leg or head motion. The microphone/loudspeaker assembly (QMC-microphone and homemade condenser loudspeaker) that picked up the echolocation calls and retransmitted the electronically modified echoes was located $30 \mathrm{~cm}$ below the bat's head.

The echolocation calls were picked up by the microphone and their frequency was electronically shifted by a controllable amount using a double heterodyning and filtering procedure (custom made device, Schuller et al. 1974). The first mixing frequency was derived from a quartz-stabilised oscillator. The frequency shift could be controlled via the adjustment of the second mixing frequency within a long term stability of $10 \mathrm{~Hz}$ (Philips PM5132 function generator). In almost all experiments the frequency shift was varied sinusoidally at different modulation frequencies and with different amounts of maximum depth. Only positive frequency shifts were presented, since the rhinolophid bats do not compensate for frequency shifts below their resting frequency.

The delay between emitted orientation calls and frequency shifted echoes corresponded to the travel time from bat to microphone and from loudspeaker to bat, and was about $1.8 \mathrm{~ms}$, equivalent to a $60 \mathrm{~cm}$ travel distance.

In order to test the interference of cluttering signals with the behaviour of Doppler shift compensation, additional acoustic stimuli were superimposed on the playback echoes. The onset of the emitted orientation call was used to trigger the broadcasting of an acoustic stimulus. Two types of interfering signals were used: pure tones and narrow band noise.

The pure tone signals could be controlled in frequency, intensity, duration and delay with respect to the onset of vocalization. The entire hearing range of the bat was tested, but experiments concentrated on the frequency range a few $\mathrm{kHz}$ below and above $\mathrm{RF}$, since only these frequencies show interference with Doppler shift compensation and cover the frequency band of possible interfering clutter in echolocation.

The narrow band noise signal was produced by multiplying a lowpass filtered noise with the desired carrier frequency. The setting of the lowpass filter cutoff frequency determined the width of the noise band which was varied between \pm 100 and $\pm 800 \mathrm{~Hz}$. The noise signal could be varied in carrier frequency, intensity, duration and delay relative to the onset of the emitted echolocation call.

The overall frequency response of the whole playback system measured from microphone input to the loudspeaker output was flat within $\pm 1 \mathrm{~dB}$ in the most relevant frequency band between 70 and $85 \mathrm{kHz}$.

The response of the bats' Doppler shift compensation system was determined from the emitted frequency of the constant frequency portion (CF) of the echolocation call. The echolocation calls were passed through a custom made frequency-to-voltage converter with a resolution of $1 \mathrm{~V} / \mathrm{kHz}$ and routed to one channel of a Watanabe Linearcorder Mark VII WR 3101. The sound pressure of the calls was determined by an AC/DC converter (homemade) and recorded in parallel on the recorder. Simultaneously the phase of the waveform modulating the second mixing frequency and thus modulating the frequency shift in the playback echo was charted on the marker channel of the recorder. 
In the undisturbed case yielding almost full compensation, the emitted frequency varies between resting frequency and a lowered frequency almost balancing the maximum frequency shift. The compensation amplitude equals the maximum frequency shift minus a compensation offset of a few $100 \mathrm{~Hz}$.

Impaired performance of Doppler shift compensation was assessed by measuring the difference between the minimum and maximum emitted frequency within each modulation cycle of Doppler shifted echoes.

Response cycles with peak-to-peak frequency differences less than half the full compensation amplitude in the undisturbed situation were considered to be distorted by the inferfering signal. Twenty or more cycles were processed for each set of test parameters. The proportion of distorted cycles to the total number of cycles in a sequence ( $\%$ interference) is used as a measure for the interference due to the cluttering signal.

\section{Results}

\section{Spontaneous vocalizations}

Bats spontaneously vocalized when they were hanging in the animal holder above the microphone/loudspeaker assembly. In these cases there was no acoustical playback. The emitted echolocation pulses were identical to those emitted under unrestrained conditions. The repetition rate of sound sequences varied between 4.2 and 17.5 pulses/s, with a mean of 7.2 pulses/s. Every 10 to $11 \mathrm{~s}$ the repetition rate of emitted sounds rose above average spontaneous rate and the average rate under experimental conditions. This rhythmical increase in repetition rate was found in all bats. It could not be correlated with any noticeable external event but might mirror rhythmic changes in the attention of the animals.

\section{Doppler shift compensation without interfering signals}

The Doppler shift compensation behaviour of the bats was tested with playback echoes. The frequency in sequences of playback echoes was modulated between the emitted frequency, i.e. zero frequency shift, to a maximum of $3 \mathrm{kHz}$ above emitted frequency in a sinusoidal time course. Except for these frequency shifts, the playback echoes were exact replicas of the emitted sounds. The bats responded to the frequency shifts of the echoes by lowering the frequency of the subsequently emitted

Table 1

\begin{tabular}{lllllll}
\hline $\begin{array}{l}\text { Modulation depth } \\
\text { BAT I }\end{array}$ & {$[\mathrm{kHz}]$} & 0.75 & 1.5 & 3.0 & 4.4 & 5.6 \\
\hline $\begin{array}{l}\text { offset } \\
\text { compens. }\end{array}$ & {$[\mathrm{Hz}]$} & 186 & 117 & 147 & 797 & - \\
$\begin{array}{l}\text { BAT II } \\
\text { offset }\end{array}$ & {$[\%]$} & 75.2 & 92.2 & 95.1 & 81.8 & - \\
compens. & {$[\mathrm{Hz}]$} & 210 & 152 & 122 & - & - \\
$\begin{array}{l}\text { BAT III } \\
\text { offset } \\
\text { compens. }\end{array}$ & {$[\mathrm{Hz}]$} & 72.0 & 89.9 & 95.9 & - & - \\
\hline
\end{tabular}

signals by an appropriate amount. As Table 1 shows all 3 specimens compensated optimally for frequency shifts up to 1.5 and $3.0 \mathrm{kHz}$. However, the Doppler shift compensation was never complete and the echo frequency which the bat heard was stabilized at the reference frequency, i.e. 110 to $250 \mathrm{~Hz}$ above the resting frequency. For frequency shifts larger than $3 \mathrm{kHz}$ the bats did not fully compensate to hold the echoes at the reference frequency. Consequently the frequency offset increased (Table 1). The Doppler shift compensation system has a slow response time (Schuller et al. 1975) and showed optimal compensation response in all 3 bats at modulation rates of $0.1 \mathrm{~Hz}$ modulation frequency.

Doppler shift compensation was operating in all 3 bats at playback intensities between $0 \mathrm{~dB}$ and $-42 \mathrm{~dB}$ relative to the sound pressure level of the emitted sound.

Doppler shift compensation with interfering signals

\section{I) Pure tones as interfering signals}

All experiments with interfering stimuli were performed under optimal conditions for Doppler shift compensation behaviour, i.e. with frequency shifts up to $3 \mathrm{kHz}$, sinusoidal modulation rates of $0.1 \mathrm{~Hz}$ and $-24 \mathrm{~dB}$ sound pressure level rel emitted SPL (except for intensity measurements).

Pure tones broadcasted to the bat concurrently with the frequency shifted playback echoes had dramatic ef-

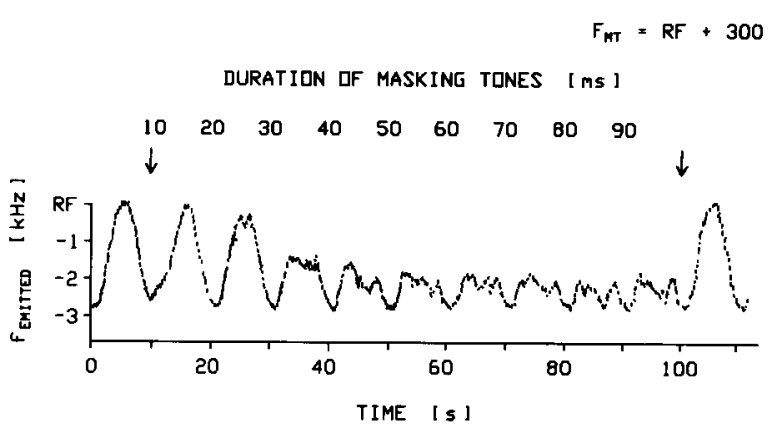

Fig. 1. Original recording demonstrating normal Doppler shift compensation and the effect of an interfering pure tone of different durations. Each dot represents the frequency of the CF portion (ordinate: emitted frequency) of consecutive echolocation pulses (abscissa: time). The playback frequency shifts are sinusoidally between 0 and $+3 \mathrm{kHz}$ at a modulation frequency of $0.1 \mathrm{~Hz}$ corresponding to a cycle time of $10 \mathrm{~s}$. The first $10 \mathrm{~s}$ show one cycle of undisturbed Doppler shift compensation starting at full compensation of almost $3 \mathrm{kHz}$, driving back to the resting frequency (frequency shift $=0$ ) and back to full compensation again. The interfering tone burst (frequency: $300 \mathrm{~Hz}$ above resting frequency, delay: $2 \mathrm{~ms}$, duration variable) is switched on at the first arrow and has little influence on shift compensation up to $20 \mathrm{~ms}$ duration. At $30 \mathrm{~ms}$ duration the interference starts to be drastic in that the emitted frequency stays at a lowered level and no longer goes back to the resting frequency, although the frequency shift travels through zero in every modulation cycle. For durations between 30 and $90 \mathrm{~ms}$ the peak-to-peak compensation amplitude difference is less than $50 \%$ of full compensation. When the interfering pure tone was switched off (arrow) the bat immediately displayed the full compensation cycle again (last cycle). 
fects on the Doppler shift compensation behaviour. Figure 1 shows a registration of unimpaired compensation behaviour (first and last modulation cycle) followed by distorted compensation when interfering tone bursts were presented. During this experimental sequence of $110 \mathrm{~s}$ the interfering pure tone of $10 \mathrm{~ms}$ duration at the beginning, was lengthened in steps of $10 \mathrm{~ms}$ and finally switched off again. Pure tones which lasted longer than 30 ms effectively interfered with Doppler shift compensation behaviour in that the bats maintained their emissions at a frequency below the resting frequency at a low level even though the frequency shifts of the echoes had decreased to zero. Frequency, duration, delay and relative intensity were parameters of the pure tones which determined the degree of interference on shift compensation behaviour.

Spectral effects: frequency of the interfering tone. Figure 2 illustrates how interference depended on the frequency of the pure tones. Doppler shift compensation behaviour was unaffected by pure tones with frequencies below resting frequency (RF) and up to $150-170 \mathrm{~Hz}$ above RF, even when the delay $(2 \mathrm{~ms})$, duration $(60 \mathrm{~ms})$ and relative intensity $(-24 \mathrm{~dB}$ re emitted intensity) were optimized for maximal interference. At pure tone frequencies $180 \mathrm{~Hz}$ above RF interference started and reached its maximum $(90 \%$ of the modulation cycles are distorted) at pure tone frequencies of $300-500 \mathrm{~Hz}$ above RF. For pure tone frequencies larger than $800-1000 \mathrm{~Hz}$ above RF interference slowly vanished.

The tuning of interference was similar in all 3 specimens, and the interference pattern for shift compensation with different maximal frequency shifts of $0.75,1.5$ and $3.0 \mathrm{kHz}$ were also almost identical (Fig. 2). These similarities allowed us to pool data among bats.

Apparently the Doppler shift compensation system is highly vulnerable to interference within a narrow fre-

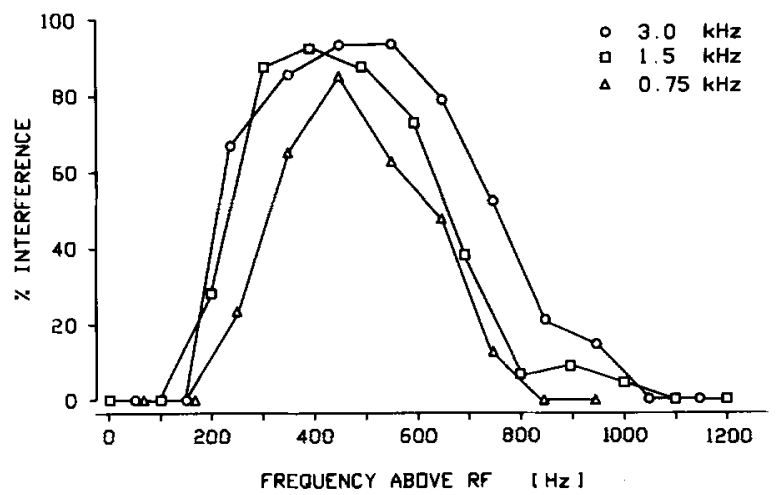

Fig. 2. Effective frequency range for pure tone interference with Doppler shift compensation. Curves indicate percentage of interference expressed as relative number of cycles with less than $50 \%$ peak-to-peak compensation amplitude for the different maximum frequency shifts $(0.75,1.5$ and $3.0 \mathrm{kHz})$. Each measuring point is derived from at least 20 cycles in each bat. Duration of the interfering pure tone was $60 \mathrm{~ms}$ and delay was $2 \mathrm{~ms}$. There is almost no interference below about $200 \mathrm{~Hz}$ and beyond $800 \mathrm{~Hz}$ above resting frequency with a maximum of interference around $400 \mathrm{~Hz}$. Maximum frequency shift has no marked influence on the pattern of the curves

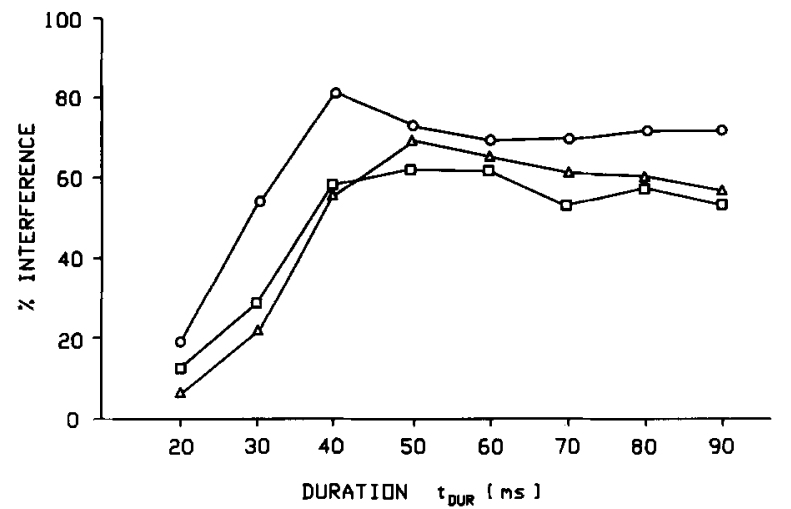

Fig. 3. Duration of the interfering pure tone. The delay of the interfering signal was at $2 \mathrm{~ms}$ after onset of the echolocation pulse and its frequency 300 to $400 \mathrm{~Hz}$ above resting frequency. Short signals below $30 \mathrm{~ms}$ have almost no effect on Doppler shift compensation. At $40 \mathrm{~ms}$ the interference reaches its maximum and stays almost constant for further increases in duration (measured up to $90 \mathrm{~ms}$ ). Curves are given for 3 animals

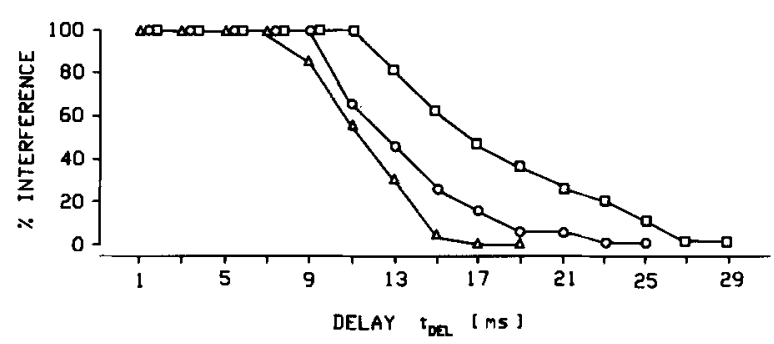

Fig. 4. Delays of interfering pure tones (frequency: 300 to 400 above RF, duration: $60 \mathrm{~ms}$ ) relative to onset of vocalization below $11 \mathrm{~ms}$ have very prominent disturbing effects on Doppler shift compensation. If the signal arrives later than $11 \mathrm{~ms}$ its interference effect drops markedly. Large delays (greater than 20-25 ms) are completely disregarded by the feedback system and have no more any effect on Doppler shift compensation. Curves are given for 3 animals

quency band centred around $450 \mathrm{~Hz}$ above RF with a width of only $600 \mathrm{~Hz}$. Signals with frequencies outside this narrow range do not disturb the Doppler shift compensation behaviour at all.

Temporal effects: duration of the interfering tone. The duration of the interfering tone is another important parameter. Interference only started at durations of at least $20 \mathrm{~ms}$, and quickly increased to a maximal level for tone durations of $40 \mathrm{~ms}$ and longer (Fig. 3). Thus, only signals which last about as long as the emitted echolocation sound effectively interfere with Doppler shift compensation behaviour.

Temporal effects: delay of the interfering tone. Pure tones maximally disturb Doppler shift compensation behaviour when they start not later than 9-11 ms after the onset of sound emission (Fig. 4). With larger delays interference declined, and in one bat any pure tone coming later than $15 \mathrm{~ms}$ after the onset of its vocalization did not affect Doppler shift compensation. Apparently, 15$25 \mathrm{~ms}$ of Doppler shifted echoes, without interference, are enough for Doppler shift compensation. 


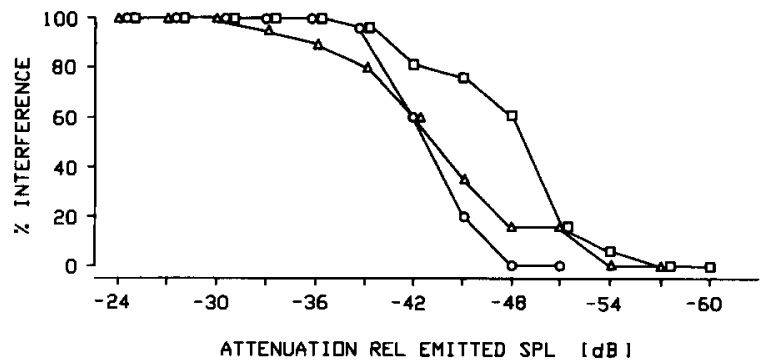

Fig. 5. Interference is efficient over a wide range of intensities $(-24$ to $-44 \mathrm{~dB}$ rel emitted sound pressure level) if the pure tone is at the optimum frequency ( $400 \mathrm{~Hz}$ above RF), is long enough (here $60 \mathrm{~ms}$ ) and arrives immediately after onset of vocalization. All 3 bats show virtually same intensity dependence of interference

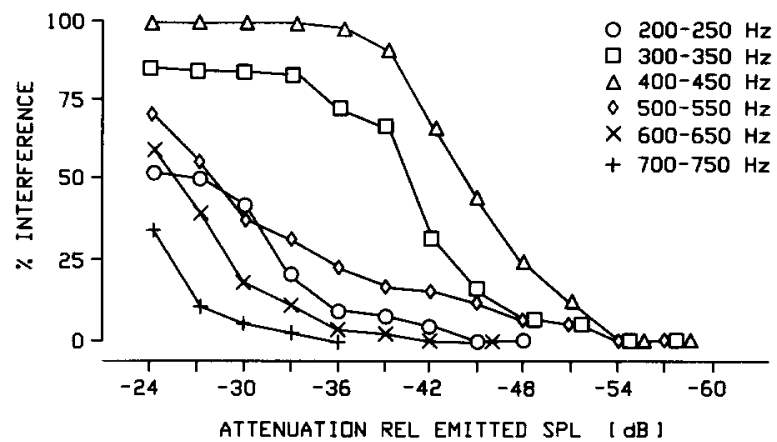

Fig. 6. Intensity dependence of interference for different pure tone frequencies. If the frequency of the pure tone lies below $300 \mathrm{~Hz}$ or on top of $500 \mathrm{~Hz}$ above RF only high intensities $(>-30 \mathrm{~dB}$ ) will induce some distortions of shift compensation

Effects of intensity of the interfering tone. Pure tones effectively interfered with Doppler shift compensation within the entire sound pressure level range of the shift compensation behaviour, which extends down to about -42 to $-50 \mathrm{~dB}$ rel SPL of emitted calls (Fig. 5). Therefore, interfering signals were already effective at threshold levels of the Doppler shift compensation behaviour, but only when the frequency of the tone was optimal for interference (about $400 \mathrm{~Hz}$ above RF). Tones with other frequencies within the narrow frequency band of interference only distorted Doppler shift compensation behaviour at high intensities (Fig. 6).

\section{II) Narrow band noise as interfering signals}

Spectral effects: carrier frequency and bandwidth of noise. As expected, only noise bands with carrier frequencies at frequencies corresponding to those of the most effective interfering tones caused maximal disturbance of the Doppler shift compensation behaviour (Fig. 7). The noise signals with a bandwidth of $\pm 100 \mathrm{~Hz}$ produced almost the same interference curves as those for pure tones, but differed in that the interference started at lower frequencies. The broader the noise band the lower was the carrier frequency at which the signal started to interfere with shift compensation. In contrast, the decline in interference for progressively higher frequencies is similar for all bandwidths and corresponded to the pattern found for pure tones.

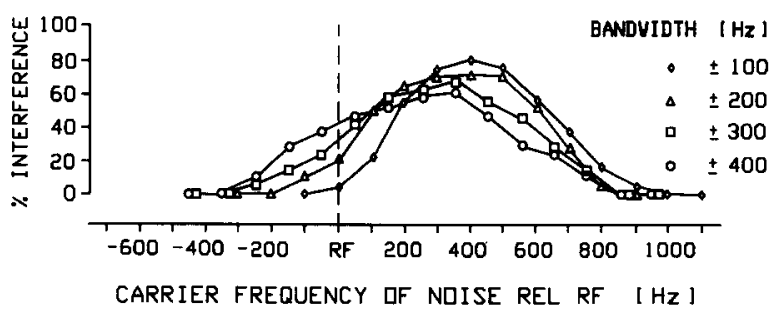

Fig. 7. Interfering effect of narrow band noise on Doppler shift compensation. The respective bandwidths are indicated on the right. Broader bandwidths lead to lower interference thresholds for the carrier frequency

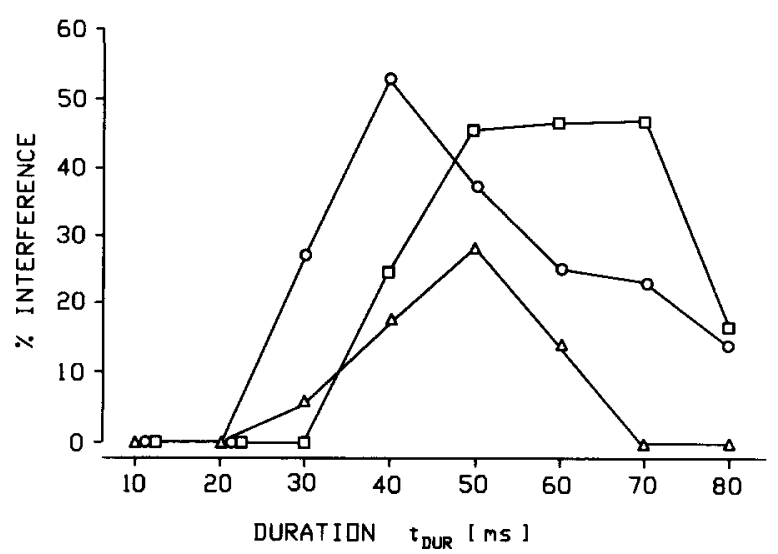

Fig. 8. Narrow band noise duration and interference with Doppler shift compensation. Only durations at and above $40 \mathrm{~ms}$ are effectively interfering with Doppler shift compensation. In contrast to pure tones, interference decreases again with increasing noise duration. Curves for 3 animals

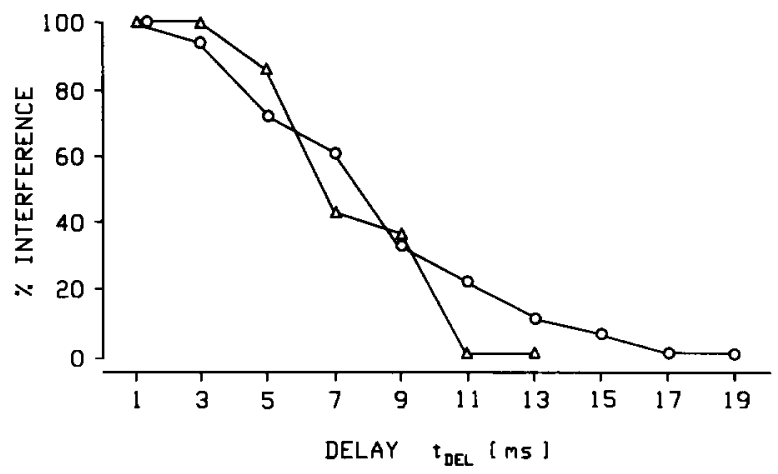

Fig. 9. Noise signal delay and its effect on compensation of Doppler shift. Only noise signals (bandwidth: \pm 200 to $300 \mathrm{~Hz}$ ) occurring almost simultaneously with echolocation calls interfere with Doppler shift compensation. Noise pulses broadcasted at delays larger than 7 to $11 \mathrm{~ms}$ after onset of vocalization do no more disturb the Doppler shift compensation system. Curves for 2 animals

Temporal effects: duration of the noise signal. As in pure tone experiments, interference reached its maximum at noise stimulus durations of $40-50 \mathrm{~ms}$ (Fig. 8). However, interference decreased again for larger durations. The duration curves were similar for all noise bandwidths tested. The interference response to noise band signals was generally less consistent compared to pure tone stim- 


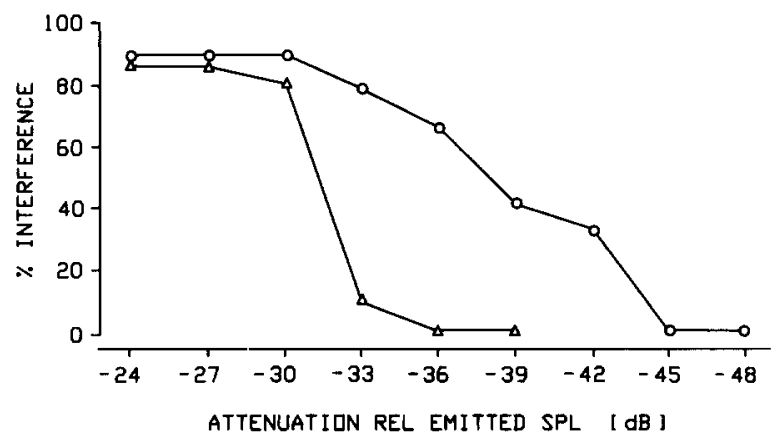

Fig. 10. Intensity of noise signal and Doppler shift compensation. Individual variations of interference curves for intensity were larger for noise signals (bandwidth: \pm 200 to $300 \mathrm{~Hz}$ ) than for pure tones and varied between the range seen in pure tones to intensity ranges extending only to about $-32 \mathrm{~dB}$ below emitted intensity. Curves for 2 animals

uli and varied more among different animals. It is not clear which mechanism is responsible for the decline of interference for longer delays.

Temporal effects: delay of the noise signal. Another dissimilarity to the interferences elicited by pure tones was found in the delay function for noise signals (Fig. 9). The disturbance already markedly declined at much shorter delays of the noise signal, i.e. at 3-5 ms after the onset of the echolocation call, compared to about $11 \mathrm{~ms}$ with pure tones. At $11 \mathrm{~ms}$ delay, the noise signals failed to influence the shift compensation. The delay function was virtually the same for all bandwidths tested.

Effects of noise intensity. The intensity was also an important parameter for the interfering effect of noise signals and showed the same principal tendency as pure tones. The intensity at which the decline of interference started was less consistent among different animals and was usually higher than in the pure tone experiments. In one animal the interference decreased abruptly at about $-32 \mathrm{~dB}$ relative to the emitted sound pressure level (Fig. 10), whereas the decline in the second animal was gradual from -32 to $-45 \mathrm{~dB}$. The pattern of intensity dependence was the same for the different noise bandwidths.

\section{Discussion}

\section{Doppler shift compensation and interference}

The stimulus situation of the present experiments was different from usual Doppler shift compensation experiments in which only the bat's signals are Doppler shifted and played back. In the present experiment interfering signals were broadcasted to the bat in addition to the Doppler shifted playback echoes. In contrast to the playback echoes, the frequency of the interfering signals was held constant and was not clamped to the emitted frequency of the bat. The playback echoes as a Doppler shifted replica of the emitted pulses always returned after a constant temporal delay of $1.8 \mathrm{~ms}$ after vocalization, whereas the superimposed interfering pure tone or noise signals were variable in delay and duration.

Even with no Doppler shifts in the playback echoes, certain conditions induced the bat to lower the emitted frequency below RF. Effective interfering signals were those with short delays, durations about that of the echolocation pulses and frequencies within 200 and $800 \mathrm{~Hz}$ above RF.

This 'compensation' behaviour induced by the interfering signals can be explained by the fact, that the command for compensation, i.e. the frequency shift above the reference frequency, persists even when the bat lowers its emitted frequency. Because the frequency of the artificial interfering echo is not clamped to the emitted frequency, the bat should decrease its emitted frequency as low as possible without reaching compensation.

In reality, the bats most frequently show an intermediate decrease of the emitted frequency as a response to the interfering signal and still display a partial compensation of the highest Doppler shifts in the playback echoes. Thus the bats vary the emitted frequency between two levels both of which are well below RF (Fig. 1).

A valid explanation for this behaviour arises from the fact that the compensation offset and therefore the reference frequency is not constant throughout the compensated Doppler shift range. Rather, the reference frequency increases with the magnitude of Doppler shift compensation (see Table 1). An interfering echo with a frequency just above reference frequency will produce a lowering of the emitted frequency, until the instantaneous reference frequency increases to match the frequency of the interfering signal. If the Doppler shifts in the playback echoes are large enough to drive the echo frequency further above the actual reference frequency, compensation will lead to further decrease of the emitted frequency.

a) Spectral parameters. The Doppler shift compensation is most efficiently disturbed by signals with frequencies 200 to $400 \mathrm{~Hz}$ above the resting frequency, and the frequency range of interference extends to about $800 \mathrm{~Hz}$ above RF. The onset of interference at about $180 \mathrm{~Hz}$ above RF coincides with the reference frequency of the bats (Schuller et al. 1974). Frequencies below the reference frequency have no interfering influence at all on the Doppler shift compensation system. The frequency band between the resting frequency and the reference frequency constitutes a kind of 'dead zone' for interference of cluttering noise. Therefore, narrow band noise signals only interfere with DSC when the upper frequency edge of the frequency band exceeds the reference frequency. Consequently, modulations superimposed on the resting frequency will not affect Doppler shift compensation as long as they fall within this dead zone.

This correlates well with results found in Rhinolophus ferrumequinum that were exposed to Doppler shifted echoes with superimposed narrow band noise signals (Schuller, unpublished). These bats always compensated 
for the upper frequency edge of the noise band, and stabilized thus the highest occurring frequency in the echo at the reference frequency.

The range of interference for pure tones extends from the reference frequency to about $600 \mathrm{~Hz}$ above reference or $800 \mathrm{~Hz}$ above RF. The interference window found for narrow band noise is very similar when referred to the upper frequency edge of the noise.

The frequency band of interference fits well into the range of best frequencies of neurons that have high Qvalues. These neurons are overrepresented in the horseshoe bat's auditory system (Schuller and Pollak 1979). The neurally overrepresented frequencies comprise a band of $1.5 \mathrm{kHz}$ in the inferior colliculus, as determined by pooling the results from several bats with slightly different reference frequencies. The band for individual bats might actually be smaller. Thus, the frequency range of interference might almost coincide with the range of overrepresented best frequencies in individual horseshoe bats.

The frequency window defined in this investigation is important for processing the constant frequency $(\mathrm{CF})$ portion of the echolocation call in Doppler shift compensation and for processing small modulations superimposed to the CF portion, e.g. fluttering target echoes. The frequency window is not important for processing the final frequency modulated (FM) portion.

Roverud (1989 b) investigated the interference of free running CF-FM signals with target ranging performance in the horseshoe bat. He found that signals within a larger frequency range disturbed the bat's ranging performance. This range was $6 \mathrm{kHz}$ below and $10 \mathrm{kHz}$ above RF and also around the lower harmonic from $6 \mathrm{kHz}$ below and $6 \mathrm{kHz}$ above $\mathrm{RF} / 2$. The frequency window defined by Roverud applies mainly to the processing of the FM frequencies which are most important for the ranging task. For this reason and because of the very different experimental conditions (high versus moderate intensities and free running versus time locked stimulus presentation), it is difficult to compare Roverud's results with ours.

b) Temporal parameters. Earlier investigations have demonstrated that proper compensation requires a minimum duration of Doppler shifted echoes and tolerates only short delays of the echoes to the onset of vocalization. Frequency shifted echoes presented outside this temporal window are completely ignored for Doppler shift compensation (Schuller 1974, 1977, 1986).

The minimum duration and maximum delay for artificial interfering signals found in this study define a similar temporal gating mechanism. Interfering signals presented outside this temporal window do not disturb shift compensation at all. Apparently, the same temporal gating mechanisms are active in Doppler shift compensation alone and during interference of pure tones or narrow band noise signals with the Doppler shift compensation system.

Roverud (1989a) explored the temporal influence of free running artificial signals on the range discrimination performance in the horseshoe bat. Only signals com- posed of CF and FM components proved to be effective in disturbing distance discrimination. FM signals alone were ineffective. However, FM sweeps alone disrupted distance discrimination effectively, if they were locked to the onset of the vocalizations and were presented within a temporal range of $28 \mathrm{~ms}$ after the end of the echolocation pulses.

Only the latter result, indicating a temporal gating process locked to the vocalization, suggests some parallels to the temporal gating conditions for Doppler shift compensation and for interference of cluttering echoes demonstrated in this study. Otherwise, it is difficult to interpret the free running case in the same terms as the temporally locked situation.

\section{Temporal and spectral gating mechanisms and clutter rejection}

Horseshoe bats are insectivorous bats that hunt for insects in acoustically dense surroundings, scrub jungle and canopy (Neuweiler et al. 1987). They encounter different types of interferences from reflections depending on the hunting strategy, i.e. whether they use the hangand-wait strategy or capture prey during flight.

When the bats are stationary in a hang-and-wait strategy, the clutter produced by their own echolocation calls returns at frequencies close to their own resting frequency but with varying delays depending on the different distances of the targets. Moving objects like leaves and branches generate a spectral broadening of the CF portion. Frequency deviations from different targets are temporally uncorrelated and randomly distributed around the resting frequency. The frequency deviations within this randomly broadened clutter band are small and will not reach the reference frequency of the bat but will fall in the dead zone of the processing system.

This clutter will not induce any Doppler compensation and therefore not shift the processing window. Only fast flying, fluttering insects or other bats will produce transient frequency deviations surmounting the small modulations of the cluttering background. These transient frequency deviations from potential prey will fall into the most sensitive frequency range and be processed appropriately. Although these large transient frequency deviations do exceed the reference frequency, they will not induce Doppler shift compensation, because of their short duration.

When the bat is flying, it will receive echoes shifted to higher frequencies due to its own flight speed. Clutter from the dense background and transient frequency shifts from potential prey are superimposed on the Doppler shifted echoes. The spectral broadening of a few $100 \mathrm{~Hz}$ due to clutter is slow and rather uniform over time, whereas the large frequency shifts created by the beating wings of an insect are transient or periodical.

The frequency transients from fluttering insects will not lead to Doppler shift compensation (Trappe and Schnitzler 1982) as they are too fast. However, the frequency components present throughout the entire duration of the temporal window of Doppler shift compensa- 
tion, i.e. the clutter induced uniform spectral broadening in the echoes from the background, will induce Doppler shift compensation. The bat compensates these Doppler shifts in a way, so that the high cutoff frequency of the narrow band clutter coincides with the reference frequency. After compensation the frequency band which is produced by the slowly moving background targets (clutter) falls in the dead zone below the reference frequency, whereas the transient large frequency deviations from fluttering insects is processed in the most sensitive frequency range above the reference frequency.

Thus the spectral and temporal gating mechanisms of the shift compensation system result in considerable enhancement of contrast between the transient signals from wing beating insects and the cluttering signals from dense background. If, in addition, high frequency shifts during glints from insect wings coincide with high echo intensity (Schnitzler et al. 1983), the signal to clutter ratio is further enhanced.

To summarize, the Doppler shift compensation system in horseshoe bats functions not only as a decoupling process keeping the receiver in its optimum working range independent of the actual flight speed of the bat, but is also an effective mechanism to reduce the disturbing clutter interference. This mechanism provides a substantial improvement of transient signal processing against a background of continuing clutter. This function is achieved by a combination of temporal and spectral gating mechanisms involved in Doppler shift compensation. These mechanisms effectively separate ongoing clutter signals from the transient signals of prey insects.

Acknowledgements. We thank G. Neuweiler and J.H. Casseday for their comments on the manuscript and A. Rau and M. Pöttke for technical assistance. Supported by the Deutsche Forschungsgemeinschaft (DFG), Sonderforschungsbereich 204 Gehör, TP 10.

\section{References}

Engelstaetter R, Vater M, Neuweiler G (1980) Processing of noise by single units of the inferior colliculus of the bat, Rhinolophus ferrumequinum. Hearing Res 3:285-300

Grinnell AD, Schnitzler HU (1977) Directional sensitivity of echolocation in the horseshoe bat. II. Behavioural directionality of hearing. J Comp Physiol 116:63-76

Neuweiler G, Metzner W, Rübsamen R, Eckrich M, Costa HH (1987) Foraging behaviour and echolocation in the rufous horseshoe bat (Rhinolophus rouxi) of Sri Lanka. Behav Ecol Sociobiol 20:53-67
Roverud RC (1989a) A gating mechanism for sound pattern recognition is correlated with the temporal structure of echolocation sounds in the rufous horseshoe bat. J Comp Physiol A 166:243249

Roverud RC (1989b) Harmonic and frequency structure used for echolocation sound pattern recognition and distance information processing in the rufous horseshoe bat. J Comp Physiol A $166: 251-255$

Roverud RC, Grinnell AD (1985a) Discrimination performance and echolocation signal integration requirements for target detection and distance determination in the CF/FM bat Noctilio albiventris. J Comp Physiol A 156:447-456

Roverud RC, Grinnell AD (1985b) Echolocation sound features processed to provide distance information in CF/FM bat Noctilio albiventris. Evidence for a gated time window utilizing both CF and FM components. J Comp Physiol A 156:457-469

Roverud RC, Grinnell AD (1985c) Frequency tracking and Doppler shift compensation in response to an artificial CF/FM echolocation sound in the CF/FM bat Noctilio albiventris. $J$ Comp Physiol A 156:471-475

Schnitzler HU (1968) Die Ultraschallortungslaute der Hufeisenfledermäuse (Chiroptera-Rhinolophidae) in verschiedenen $\mathrm{Or}$ ientierungssituationen. Z Vergl Physiol 57:376-408

Schnitzler HU, Grinnell AD (1977) Directional sensitivity of echolocation in the horseshoe bat, I. Directionality of sound emission. J Comp Physiol 116:51-61

Schnitzler HU, Menne D, Kober R, Heblich K (1983) The acoustical image of fluttering insects in echolocating bats. In: Huber F, Markl H (eds) Neuroethology and behavioural physiology. Springer, Berlin Heidelberg New York, pp 235-250

Schuller G (1974) The role of overlap of echo with outgoing echolocation sound in the bat. Rhinolophus ferrumequinum. Naturwissenschaften $61: 171-172$

Schuller G (1977) Echo delay and overlap with emitted orientation sounds and Doppler-shift compensation in the bat, Rhinolophus ferrumequinum. J Comp Physiol 114:103-114

Schuller G (1980) Hearing characteristics and Doppler shift compensation in south Indian CF-FM bats. J Comp Physiol $139: 349-356$

Schuller G (1986) Influence of echolocation pulse rate on Doppler shift compensation control system in the greater horseshoe bat. J Comp Physiol A 158:239-246

Schuller G, Beuter K, Schnitzler HU (1974) Response to frequency shifted artificial echoes in the bat, Rhinolophus ferrumequinum. J Comp Physiol 89:275-286

Schuller G, Beuter K, Rübsamen R (1975) Dynamic properties of the compensation system for Doppler-shifts in the bat, Rhinolophus ferrumequinum. J Comp Physiol 97:113-125

Schuller G, Pollak GD (1979) Disproportionate frequency representation in the inferior colliculus of Doppler-compensating greater horseshoe bats: evidence for an acoustic fovea. J Comp Physiol 132:47-54

Schuller G, Suga N (1976) Laryngeal mechanisms for the emission of CF-FM sounds in the Doppler-shift compensating bat, Rhinolophus ferrumequinum. J Comp Physiol 107:253-262

Trappe M, Schnitzler HU (1982) Doppler-shift compensation in insect-catching Horseshoe bats. Naturwissenschaften 69:193194 